\title{
Erratum
}

\section{Melting Pressure Thermometry of the Saturated Helium Mixture at Millikelvin Temperatures}

\author{
E. Pentti, ${ }^{1}$ J. Tuoriniemi, ${ }^{1}$ A. Salmela, ${ }^{1}$ and A. Sebedash ${ }^{1,2}$ \\ ${ }^{1}$ Low Temperature Laboratory, P.O. Box 2200, TKK 02015, Finland \\ E-mail: epentti@cc.hut.fi \\ ${ }^{2}$ P. L. Kapitza Institute for Physical Problems, Kosygin Str. 2, Moscow, Russia
}

Erratum to: Journal of Low Temperature Physics, Vol. 146, Nos. 1/2, January 2007, p. 71-83

DOI: $10.1007 / \mathrm{s} 10909-006-9267-8$

The incorrect metadata for this article was inadvertently posted online. The correct metadata is as follows:

Melting Pressure Thermometry of the Saturated Helium Mixture at Millikelvin Temperatures

E. Pentti, ${ }^{1}$ J. Tuoriniemi, ${ }^{1}$ A. Salmela, ${ }^{1}$ and A. Sebedash ${ }^{1,2}$

${ }^{1}$ Low Temperature Laboratory, P.O. Box 2200, TKK 02015, Finland e-mail: epentti@cc.hut.fi

2 P. L. Kapitza Institute for Physical Problems, Kosygin Str. 2, Moscow, Russia

The melting pressure of $a^{3} \mathrm{He}^{4}{ }^{4} \mathrm{He}$ mixture has a very simple quadratic temperature dependence below some tens of $m K$, determined by the entropy of the ${ }^{3}$ He component in the liquid mixture. For undersaturated mixtures, the melting pressure also depends on the ${ }^{3}$ He concentration $x$, which may vary in the course of the experiment as ${ }^{4} \mathrm{He}$ transfers between the liquid and the solid phases. On the other hand, if the mixture is saturated, the system is in a univariant state with a melting pressure that depends uniquely on temperature and, thus, offers a thermometric standard. However, the univariant state

The online version of the original article can be found at http://dx.doi.org/10.1007/s10909-006-9267-8 
includes a pure liquid ${ }^{3}$ He phase, which complicates the temperature dependence around its superfluid transition temperature $T_{\mathrm{c}}$. In this paper, we analyze the melting pressure of the saturated mixture in simple terms and find an expression that is in good agreement with our experimental data, and is applicable across $T_{\mathrm{c}}$ own to very low temperatures. The obtained derivatives of the melting pressure with respect to the square of temperature are $0.92 \mathrm{~Pa}$ $\leq m K^{-2}$ above $T_{\mathrm{c}}$ and $1.52 \mathrm{~Pa} \leq m K^{-2}$ in the zero-temperature limit.

PACS Numbers: 67.57.Bc; 67.60.Fp; 67.80.Gb.

\section{REFERENCES}

1. R. L. Rusby et al., J. Low Temp. Phys. 126, 633-642 (2002).

2. Yu. D. Anufriev, V. N. Lopatik, and A. P. Sebedash, JETP Lett. 37, 45-47 (1983); V. N. Lopatik, Sov. Phys. JETP 59, 284-289 (1984).

3. A. P. Sebedash, JETP Lett. 65, 276-281 (1997).

4. B. van den Brandt et al., Physica 114B, 295-306 (1982).

5. G. E. Watson, J. D. Reppy, and R. C. Richardson, Phys. Rev. 188, 384 (1969).

6. M. Kollar and D. Vollhardt, Phys. Rev. B 61, 15347 (2000).

7. A. Driessen and E. van der Poll, Phys. Rev. B 33, 3269 (1986).

8. E. Tanaka, K. Hatakeyama, S. Noma, and T. Satoh, Cryogenics 40, 365-377 (2000).

9. E. Krotschek, M. Saarela, K. Schörkhuber, and R. Zillich, Phys. Rev. Lett. 80, 4709 (1998).

10. D. S. Greywall, Phys. Rev. B 33, 7520 (1986).

11. A. P. Sebedash et al., in Proceedings of QFS 2006, J. Low Temp. Phys, to be published.

12. A. Sebedash et al., AIP Conf. Proc. 850, 1591 (2006).

13. S. Balibar, H. Alles, and A. Parshin, Rev. Mod. Phys. 77, 317-370 (2005). 